\title{
Desempeño de Capitán de la Sabana (Eremophilus mutisii) en estadios tempranos de desarrollo
}

\section{Performance of the Captain of the Savannah, (Eremophilus mutisii) in early stages of development}

\author{
Erika Moncaleano-Gómez ${ }^{1}$; Camilo Prieto-Mojica²
}

\begin{abstract}
'Zootecnista, M.Sc. Universidad de Ciencias Aplicadas y Ambientales U.D.C.A, Facultad de Ciencias Agropecuarias. Bogotá, D.C., Colombia; e-mail: erikamarcelamg@gmail.com; (D) https://orcid.org/0000-0001-6807-2877

ZZootecnista, M.Sc. Ph.D. Universidad de Ciencias Aplicadas y Ambientales U.D.C.A, Facultad de Ciencias Pecuarias. Bogotá, D.C., Colombia; e-mail: camprieto@udca.edu.co; D https://orcid.org/0000-0002-4987-3596
\end{abstract}

Cómo citar: Moncaleano-Gómez, E.; Prieto-Mojica, C. 2020. Desempeño de Capitán de la Sabana (Eremophilus mutisii) en estadios tempranos de desarrollo Rev. U.D.C.A Act. \& Div. Cient. 23(2):e1216. http://doi.org/10.31910/rudca.v23.n2.2020.1216

Artículo de acceso abierto publicado por Revista U.D.C.A Actualidad \& Divulgación Científica, bajo una licencia Creative Commons CC BY-NC 4.0

Publicación oficial de la Universidad de Ciencias Aplicadas y Ambientales U.D.C.A, Institución de Educación Superior Acreditada de Alta Calidad por el Ministerio de Educación Nacional.

Recibido: Julio 14 de 2020 Aceptado: Diciembre 4 de 2020 Editado por: Ingeborg Zenner de Polanía

\section{RESUMEN}

Son varios los esfuerzos realizados en el pez capitán de la sabana Eremophilus mutisii para su uso y conservación; sin embargo, a pesar de los avances logrados, no se ha conseguido superar las dificultades de la larvicultura en esta especie, razón por la cual, se realizó la primera descripción de las etapas iniciales de vida, con el fin de contribuir y de mejorar los inicios de la larvicultura. Las larvas utilizadas fueron obtenidas de reproductores maduros de capitán de la sabana. Posteriormente, se procedió a describir el volumen y determinación de absorción del saco vitelino, la abertura máxima de la bucal, primera alimentación, preferencia alimentaria y la evaluación de variables productivas, como ganancia de peso, tasa de crecimiento específica y sobrevivencia. El consumo del saco vitelino, se completó en la 201 Hora Post Eclosión (HPE), equivalente a más del $90 \%$ de absorción, la apertura máxima bucal es alcanzada a las $126 \mathrm{HPE}$. Esta especie presenta un alto de grado de aceptación a la alimentación con quistes de artemia, a partir del 11 Día Post Eclosión(DPE) y, posteriormente, a alimento artificial, desde los $47 \mathrm{DPE}$; lo anterior, se convierte en un aporte para la obtención e identificación de mecanismos y estrategias que promuevan el uso y la conservación en cautiverio de E. mutisii, con el fin de fomentar el aprovechamiento acuícola en este especie, que se encuentra en amenaza de extinción y puede ser una fuente alimenticia, que contribuya a la seguridad alimentaria de la población Cundiboyacense.

Palabras clave: Pez nativo; Cautiverio; Capitán de la Sabana, Trichomycteridae.

\section{ABSTRACT}

There are several efforts made in the savannah captain fish Eremophilus mutisii for its use and conservation; however, despite the progress made, it has not been possible to overcome the difficulties of larviculture in this species, reason for to make the first description 
of the initial stages of life for contribute and improve the beginnings of larviculture. The larvae used were obtained from mature savanna captain breeders. Subsequently, the volume and determination of the absorption of the yolk sac, the maximum opening of the mouth, first feeding, food preference and the evaluation of productive variables such as: weight gain, specific growth rate and survival were described. The consumption of the yolk sac was completed at 201 Hour Post Hatching (HPE), equivalent to more than 90\% absorption, the maximum mouth opening is reached at $126 \mathrm{HPE}$. This species has a high degree of acceptance when feeding with artemia cysts from 11 Day post-hatching (DPE) and later to artificial food from 47 DPE, This to becomes a contribution to the obtaining and identification of mechanisms and strategies that promote the use and conservation of E. mutisii in captivity, in order to promote the use of aquaculture in this species that is threatened with extinction and can be a food source that contributes to the food security of the Cundiboyacense population.

Keywords: Native fish; Captivity; Captain of the Savannah, Trichomycteridae.

\section{INTRODUCCIÓN}

Una de las finalidades de la larvicultura es incrementar las tasas de sobrevivencia en la transición de la etapa larvaria a la etapa juvenil, a partir de manejo de condiciones ambientales adecuadas, como temperatura, calidad del agua y establecimiento de estrategias alimentarias, que garanticen una producción de alevinos, continua y estable (García et al. 2006). Por lo tanto, la indagación en el desarrollo larval de los peces es de gran importancia para desarrollar sistemas de producción en especies de interés acuícola, como el capitán de la sabana Eremophilus mutisii, el cual, ha sido considerado como una especie de aguas frías excepcional, presentando potencial para la producción piscícola (Rosado, 2007; Landines et al. 2017).

Dentro de la fauna dulceacuícola más representativa y emblemática del río Bogotá y sus afluentes, se encuentra el capitán de la sabana. Desde tiempos ancestrales, este pez era importante en la región del altiplano Cundiboyacense porque formaba parte de la dieta de los indígenas nativos y, en la actualidad, hace parte del sustento alimenticio de los pobladores (Lemus-Portillo et al. 2017; Moreno et al. 2019).

Las acciones humanas desarrolladas en los últimos tiempos han causado un deterioro en los cuerpos de agua y ecosistemas aledaños, generando afectaciones en las poblaciones de E. mutisii, al disminuir los individuos de la especie, hasta tal punto que, entre el 2002 y el 2012, pasó de estar en la clasificación de la UICN, como "Casi Amenazada" (Mojica et al. 2002) a "Vulnerable" (Mojica et al. 2012). Esto indica, la importancia de enfocar esfuerzos hacia un trabajo concienzudo en dicha especie, para poder recuperar un estado poblacional ideal (Lemus-Portillo et al. 2020).

Debido a lo descrito anteriormente, en varias reuniones científicas, se ha reiterado la importancia de adelantar trabajos que permitan incorporar la especie a sistemas productivos en cautiverio
(Valderrama et al. 2011), dado que algunos autores, la han catalogado como la única especie nativa candidata de aguas frías, con posibilidad de ser trabajada en piscicultura (Moreno et al. 2019).

Los estudios biológicos en embriones, larvas y post larvas de peces son determinantes para identificar las características de crecimiento, desarrollo, alimentación, comportamiento, sobrevivencia y nutrición, siendo la última, la mayor limitante para la producción acuícola (Gore, 2006).

La primera alimentación es uno de los aspectos claves y fundamentales para la sobrevivencia y el crecimiento de las especies. Permitir que las post larvas se alimenten, luego la absorción del saco vitelino, depende de varios factores, como el tamaño de la presa, la apertura bucal, el tipo de nado, la capacidad visual y la cantidad de alimento disponible para cada larva (Hamre et al. 2008).

Adicionalmente, el estudio de las relaciones longitud-peso suministran referentes indirectos acerca de las etapas de crecimiento, desarrollo y nutrición, por ende, del estado de salud de las poblaciones (Arismendi et al. 2011). Por lo tanto, la finalidad del presente estudio, se centró en describir el desarrollo y el desempeño en estadios tempranos del capitán de la sabana, a través de procedimientos morfológicos.

\section{MATERIALES Y MÉTODOS}

Localización y descripción del área de estudio: Los procesos de reproducción y larvicultura fueron desarrollados en la Unidad Académica El Remanso, perteneciente a la Universidad de Ciencias Aplicadas y Ambientales U.D.C.A, sede Bogotá, Colombia, que se encuentra ubicada a $2.600 \mathrm{~m}$ s.n.m y temperatura media entre $9 \mathrm{y}$ $20^{\circ} \mathrm{C}$ (IDEAM, 2016).

Material biológico: Las larvas utilizadas en el presente estudio fueron obtenidas de reproductores maduros de capitán de la sabana, transportados desde la represa del Sisga, ubicada en el municipio de Choncontá, Cundinamarca N5 $5^{\circ} 05^{\prime} 17$, W7 $73^{\circ} 43^{\prime} 18 \mathrm{~W}$. Los parámetros físico-químicos encontrados en esta represa fueron: Alcalinidad: 13,4mg $\mathrm{CaCO}_{3} / \mathrm{L}$; Oxígeno Disuelto: 7,6mg/L; pH: 7,4 y Temperatura: $15^{\circ} \mathrm{C}$.

Para la recepción de los animales en el laboratorio, se contó con cuatro tanques de 250 litros de agua, cada uno, provistos de aireación constante, en un sistema cerrado de recirculación de agua, con el fin de generar adaptación ambiental de los reproductores al cautiverio y, posteriormente, realizar el protocolo de inducción hormonal, recomendado por González-Acosta \& Rosado-Puccini (2005), con algunas modificaciones: Primera dosis hembra: $0,25 \mathrm{mg} / \mathrm{kg}$ de peso de la hembra; segunda dosis hembra: $0,5 \mathrm{mg} / \mathrm{kg}$ de peso de la hembra (24 horas después de la primera dosis); tercera dosis hembra: $5 \mathrm{mg} / \mathrm{kg}$ de peso de la hembra (12 horas después de la segunda dosis). Dosis única machos: $4 \mathrm{mg} / \mathrm{kg}$ de peso de cada macho (a la misma hora de la tercera dosis de la hembra). El número de animales utilizados en el protocolo de reproducción fue de 3 machos por 2 hembras. 
Después de 24 horas de la última aplicación hormonal, los ovocitos fueron obtenidos por presión abdominal y fertilizados con el semen, activando los gametos con agua y permitiendo la hidratación de los óvulos. Posteriormente, fueron llevados a las incubadoras, tipo bandeja, sugeridas por Moncaleano et al. (2018), a una temperatura promedio de $13^{\circ} \pm 1^{\circ} \mathrm{C}$.

Las larvas eclosionaron a las 72 horas post fertilización (HPF), debidamente contadas y sembradas en tres acuarios de $40 \mathrm{~L}$, a densidades de 10 larvas $/ \mathrm{L}$ y temperatura promedio de $15,7 \pm 0.4^{\circ} \mathrm{C}$, $6,4 \mathrm{mg} / \mathrm{L}$ de oxígeno disuelto y $\mathrm{pH}$ de 8.0. Los acuarios fueron cubiertos con plástico negro, con el fin de disminuir intensidad lumínica.

Toma de muestras. Para las biometrías, se siguió el protocolo de bienestar animal, sacrificando los individuos en sobredosis de MS 222 (200ppm) y fijados en formol bufferado al 4\%. Se tomaron 10 individuos por muestra, desde la 6 hora post eclosión (HPE), hasta la $342 \mathrm{HPE}$, con intervalos de 12 horas.

Volumen de Saco Vitelino. Para el volumen del saco vitelino, se utilizaron fórmulas de medición esférica $\left(V_{1}=4 / 3 \varpi r^{3}\right)$ y elipsoidal $\left(\mathrm{V}_{2}=\left[(\varpi / 6) * \mathrm{~L}^{*} \mathrm{H}^{2}\right]\right)$, donde $\mathrm{V}$, es el volumen del saco vitelino $\left(\mathrm{mm}^{3}\right)$, $r^{3}$ es el promedio de la medida de altura y longitud del saco vitelino muestreado, elevado al cubo (Aristizabal, 2006), "L", la longitud total del saco vitelino ( $\mathrm{mm}$ ) y " $\mathrm{H}$ ", la altura del saco vitelino ( $\mathrm{mm})$ (Valbuena V. et al. 2013). Para la medición de estos parámetros, se utilizó el programa Image Pro Plus, versión 4.1.

Determinación de la absorción del saco vitelino. Aplicando el procedimiento anterior, se calculó el porcentaje de absorción del saco vitelino (RSV), mediante la fórmula $\mathrm{RSV}=\mathrm{Vm} * 100 / \mathrm{Vi}$, donde $\mathrm{Vm}$ es el volumen del saco vitelino $\left(\mathrm{mm}^{3}\right)$, a la hora del muestreo y Vi es el volumen del saco vitelino $\left(\mathrm{mm}^{3}\right)$, de las larvas recién eclosionadas (Valbuena V. et al. 2013).

Abertura máxima de la bucal (AMB). La medición de la abertura máxima bucal (AMB), se realizó considerando un ángulo de $90^{\circ}$, según la fórmula $\mathrm{AMB}=\mathrm{LMS} \times \sqrt{2}$, donde, LMS corresponde a la longitud máxima del maxilar ( $\mu \mathrm{m})$ (Valbuena V. et al. 2013).

Primera alimentación y preferencia alimenticia. Cuando las larvas presentaron 50\% de absorción del saco vitelino, se dio inicio a la alimentación exógena con quistes $(200 \mu \mathrm{m})$ y nauplios recién eclosionados de artemia $(350-450 \mu \mathrm{m})$, para determinar la abertura bucal y preferencia alimenticia.

Seguido a esta etapa, para la preferencia alimenticia, se seleccionaron al azar 10 larvas/L y se colocaron en recipientes de vidrio, alimentadas con 100 organismos por larva, que correspondían a quistes - nauplios de artemia y micro gusano de avena (Panagrellus redivivus), en iguales proporciones, hasta el 47 día post eclosión (DPE), momento, en el cual, se dio inicio a la alimentación con alimento balanceado molido, en partículas menores a $1,5 \mathrm{~mm}$ y se procedió a la toma de muestras de 20 larvas, sacrificadas con MS 222 (200ppm) y fijadas en formol bufferado al 4\%, con el fin de analizar el contenido intestinal y, de esta manera, identificar la preferencia alimenticia.

Evaluación de variables productivas. Una vez determinada la preferencia alimenticia, se evaluó la ganancia diaria de peso, mediante la fórmula: Pf-Pi/ número de días, donde, Pf corresponde al peso final y Pi, al peso inicial (Torres-Tabares et al. 2014).

La tasa de crecimiento específico, se evaluó en talla y en peso, mediante las fórmulas: T.C.E $(\% /$ día $)=$ LnTf-LnTi/Número de días* 100, donde, T.C.E es tasa de crecimiento específica, LnTf, logaritmo natural de la talla final y LnTi, logaritmo natural de la talla inicial (Arce-Uribe \& Luna-Figueroa, 2003).

T.C.E $(\% /$ día $)=$ LnPf-LnPi/Número de días* 100, donde, LnTf es el logaritmo natural del peso final y $\mathrm{LnPi}$, logaritmo natural del peso inicial (Arce-Uribe \& Luna-Figueroa, 2003). Por último, se evaluó la sobrevivencia, mediante la fórmula: $(\mathrm{Nf} / \mathrm{Ni}) * 100$, donde, $\mathrm{Nf}$ es el número de individuos sobrevivientes al final del periodo de tiempo y Ni, número inicial de individuos (Arce-Uribe \& Luna-Figueroa, 2003; Valbuena V. et al. 2013).

\section{RESULTADOS Y DISCUSIÓN}

Etapa Larval. El saco vitelino del E. mutisii tiene dos fases de absorción: la primera comprende desde las 6 hasta las 96 Horas Post Eclosión (HPE), donde el saco vitelino presenta forma esférica. Posteriormente, la segunda fase comprende de la 102 hasta la 201 HPE, en donde el saco vitelino presenta forma elipsoidal.

De acuerdo con las mediciones en las muestras realizadas, el consumo del saco vitelino empezó a partir de la $6 \mathrm{HPE}$ y se completó en la $201 \mathrm{HPE}$, equivalente a más del 90\% de absorción, con 9 días de edad de la larva (Figura 1 y 2).

Las dos tasas de absorción mencionadas concuerdan, parcialmente, con la primera fase post-eclosión, esférica en E. mutisii, que se caracteriza por una tasa de absorción de vitelo, relativamente constante (Li \& Mathias, 1982; Quantz, 1985). En la segunda fase post-eclosión, se terminan de consumir las reservas vitelinas de una manera más lenta, lo cual, hace que la tasa de absorción disminuya, este patrón de consumo, lo presentan especies, como Lutjanus campechanus (Williams et al. 2004) y Pagrus pagrus (Aristizabal, 2006); sin embargo, en esta segunda fase, E. mutisii presentó un saco vitelino en forma elipsoidal y una absorción más acelerada (Figura 2). Se resalta que el consumo del saco vitelino fue constante durante este periodo y no presentó alteraciones durante el proceso.

En E. mutisii, se observan remanentes de vitelo hasta las $201 \mathrm{HPE}$. Este resultado difiere de otras especies, como Dormitator latifrons y Pseudoplatystoma punctifer, en donde la absorción es más rápida (Gisbert et al. 2014; López-López et al. 2015); esta tasa de absorción es importante para el crecimiento, la supervivencia de las larvas y el desarrollo inicial, en general. La velocidad de estos eventos, se puede ver afectada por factores genéticos y ambientales (Falk-Petersen, 


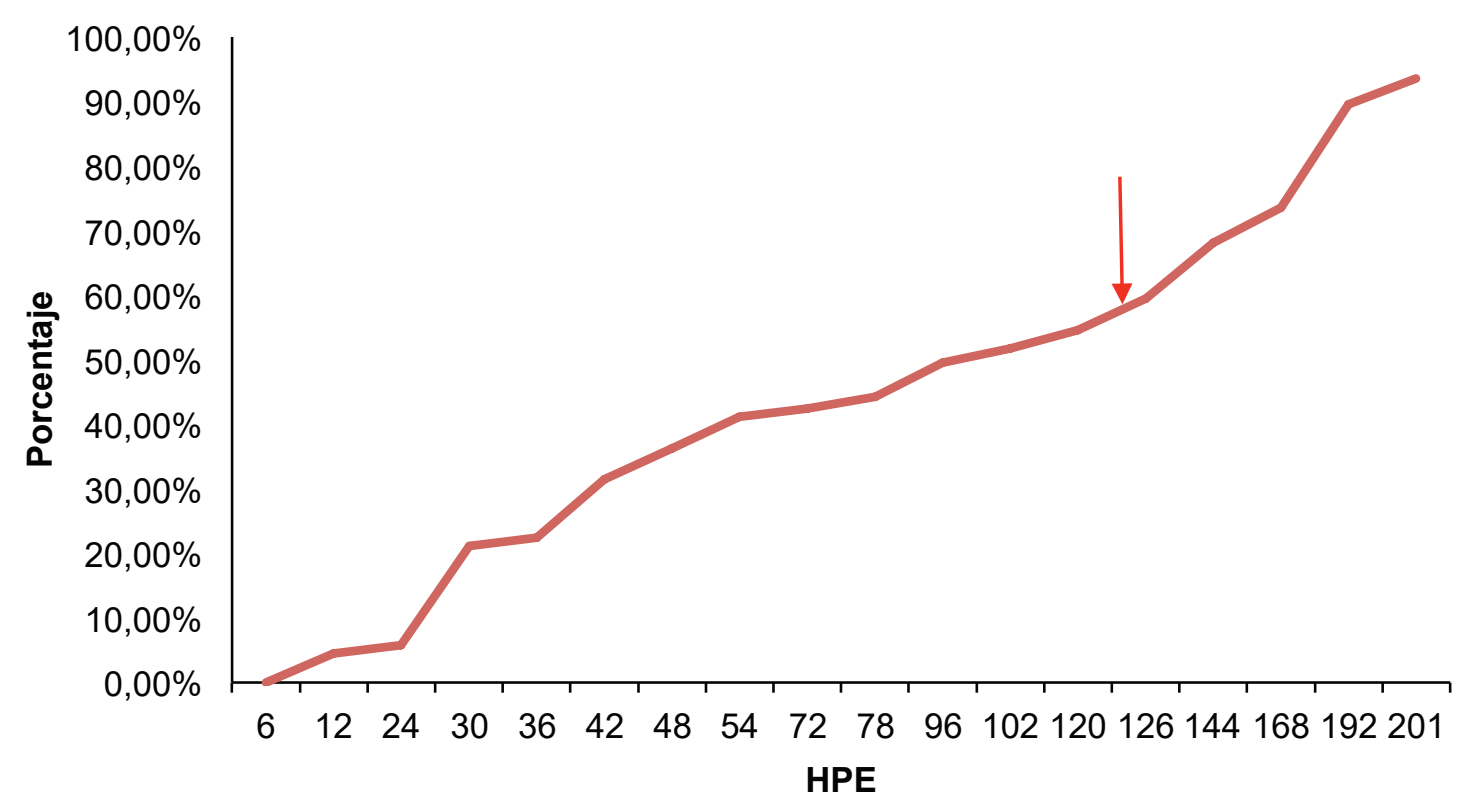

Figura 1. Absorción del saco vitelino de E. mutisii en los primeros 9 días PE. Flecha roja indica abertura máxima bucal a las 126 HPE.

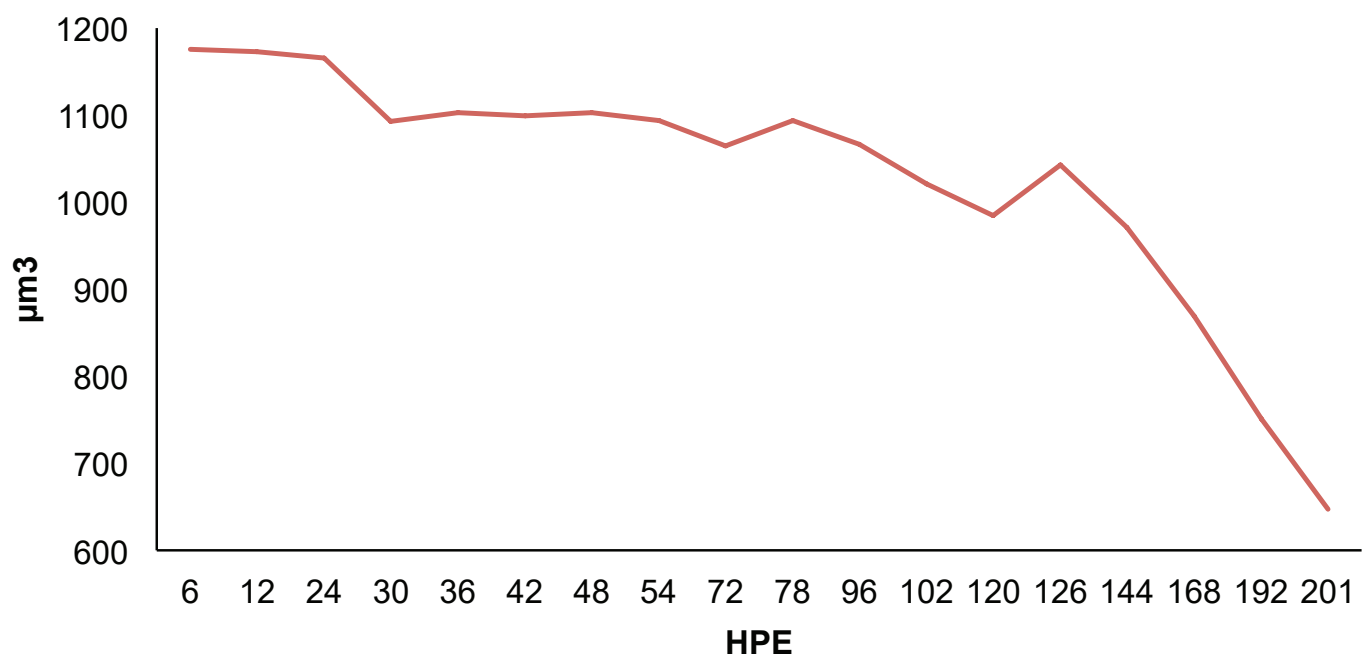

Figura 2. Volumen del saco vitelino de E. mutisii durante el proceso de absorción. 9 días PE.

2005) que, principalmente, están asociados a parámetros de agua, como oxígeno y temperatura, además de la intensidad lumínica. $\mathrm{Al}$ ser E. mutisii una especie de agua fría, muestra una absorción del saco vitelino semejante a la de la Trucha arco iris Oncorbynchus mykiss (FAO, 2009), lo que indica que la temperatura juega un papel importante en esta absorción.

E. mutisii muestra una apertura bucal, a nivel macroscópico, a partir de las $72 \mathrm{HPE}$; sin embargo, según las mediciones realizadas, la apertura máxima bucal (AMB) es alcanzada a las $126 \mathrm{HPE}$, con una

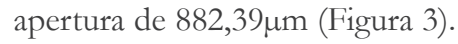

Comparado con especies, como el Pterophyllum altum, que presenta a las 72 HPE su AMB (Torres-Tabares et al. 2014), el E. mutisii presenta una apertura bucal tardía, hasta las $126 \mathrm{HPE}$, lo cual, muestra que el inicio de la alimentación exógena se realiza con remanentes de vitelo y esto puede ser dado por la maduración de desarrollo en el tracto gastrointestinal de la especie, en donde combina la alimentación endógena con la exógena desde las $126 \mathrm{HPE}$, hasta las $201 \mathrm{HPE}$. Este momento es importante desde el punto de vista productivo, pues a partir de la $126 \mathrm{HPE}$, se logró iniciar la alimentación exógena. 


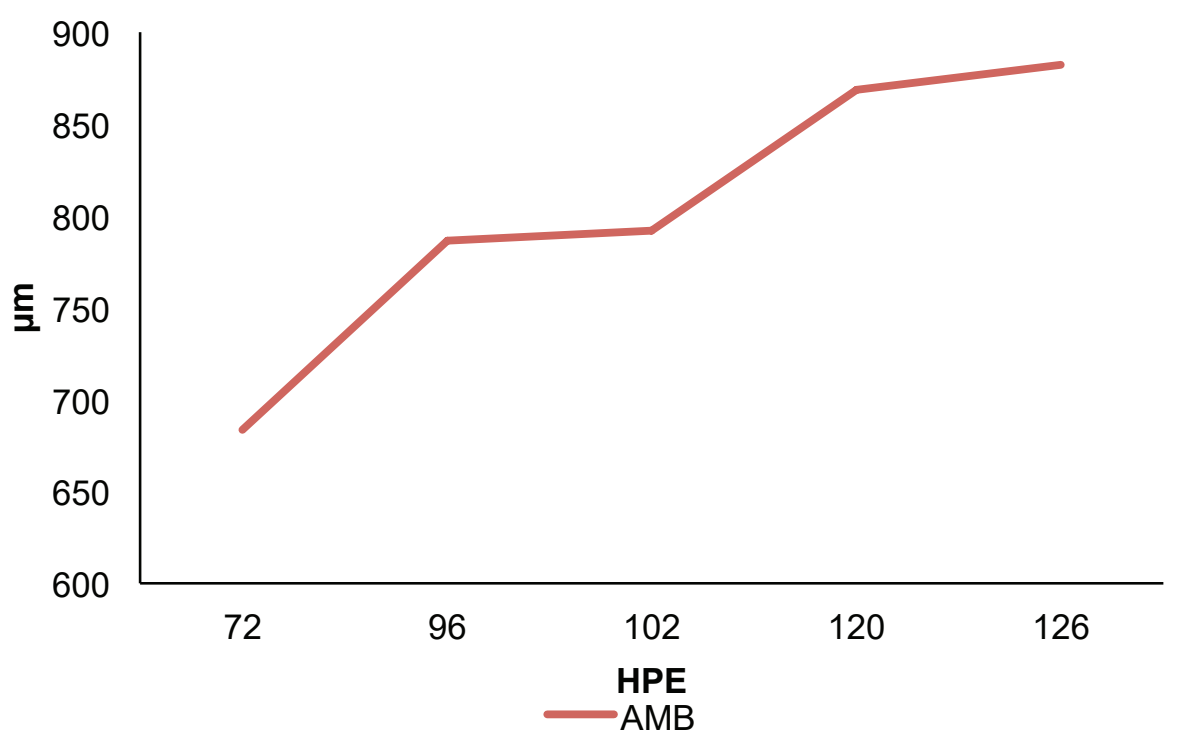

Figura 3. Abertura Máxima Bucal AMB (126 HPE).

La larva de E. mutisii presenta una longitud total de $3,7 \mathrm{~mm}$ a los 6 HPE y a los 9 días PE, presenta una longitud total de $6,88 \mathrm{~mm}$; lo anterior, está influenciado por las condiciones físico químicas del aguak principalmente.

Post Larva. A partir del 11 DPE (día post eclosión), se empezó a suministrar Artemia salina y micro gusano de la avena (Panagrelus redivivus), con una frecuencia de 3 veces al día. Hasta el 14 DPE, no fue posible evidenciar el contenido estomacal, debido a que el estómago, en esta etapa, no se encontraba aún desarrollado y fue a partir del 14 DPE, que se logró evidenciar en el contenido intestinal de la larva, el consumo de quistes de artemia, de manera macroscópica y microscópica (Figura 4); lo anterior, permitió identificar la preferencia alimenticia de consumo de la post larva, por los quistes de artemia.
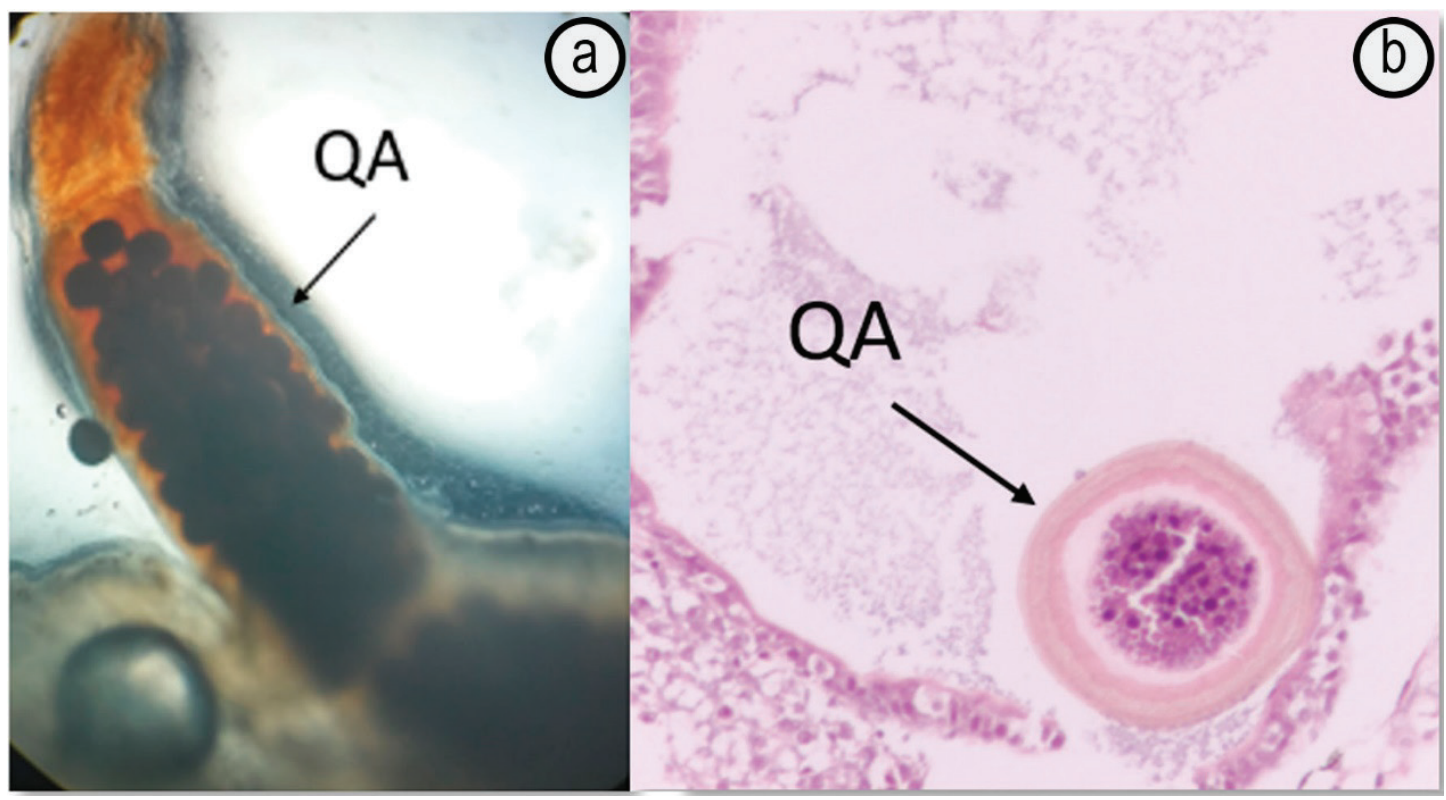

Figura 4. Contenido intestinal de post larva 14 DPE. QA: Quistes de Artemia. a. 40x; b. 200x H\&E. 
La sobrevivencia en esta etapa del E. mutisii fue del 58,5\%, semejante

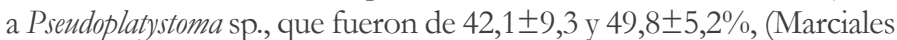
et al. 2010). En silúridos, se ha observado que las principales causas de mortalidad están relacionadas con canibalismo, que puede ser resultado de su comportamiento alimenticio agresivo, altas densidades y el manejo, debido al escaso conocimiento de su comportamiento natural (Kestemont et al. 2003; Atencio \& Zaniboni, 2006); sin embargo, en E. mutisii, ningún momento se evidenció canibalismo.

En sistemas de producción y en estudios nutricionales acuícolas, se ha identificado que los tipos de alimentos proporcionados a los peces influyen directamente en la tasa de crecimiento, indicadora de la calidad proteica de las dietas que, a su vez, es influenciada por la proporción de aminoácidos esenciales suministrados, que se evidencian en la ganancia en peso (Tacon, 1987; Soriano Salazar \& Hernández Ocampo, 2002).

Alevino. A partir del 47 DPE, se inició la co-alimentación, que consistió en suministrar $A$. salina y alimento artificial comercial para peces ornamentales $(360-560 \mu \mathrm{m})$, cuya característica nutricional fue de 49,9\% de proteína total; 6,3\% de grasa; 0,64\% de fibra cruda, 9,25 de ceniza y 4,3\% de humedad. A partir de este día 47, se comenzó a observar el consumo de este alimento, microscópicamente, en el intestino.

A partir del 50 DPE, se suspendió la alimentación con artemia y se suministró únicamente alimento artificial a voluntad, debido a que se observaba un comportamiento activo en los animales por consumir este alimento, ofrecido cuatro veces al día.

La sobrevivencia en esta etapa de alevinaje, comprendida desde el 47 DPE hasta el 77 DPE, fue de 89,54\%; adicionalmente, la ganancia de peso fue de $0,012 \mathrm{~g} /$ día y la tasa de crecimiento específica fue de $5,44 \%$, en peso y $2,95 \%$, en longitud (Figura 5).
La ganancia de peso para E. mutisii fue de $11,45 \mathrm{mg} /$ día, distinta a la del Ictalurus balsanus, que fue de $23,22 \mathrm{mg} /$ día (Arce-Uribe \& Luna-Figueroa, 2003), aunque los tiempos del estudio para ambas especies, no fueron los mismos.

En el caso de otros bagres, como Pseudoplatystoma sp. y Leiarius marmoratus, alimentados con $A$. salina, han presentado mayor ganancia de peso y talla, que en larvas alimentadas con otro tipo de alimento (Marciales et al. 2010); sin embargo, las tasas de crecimiento del E. mutisii pueden llegar a ser más altas con un alimento de mayor digestibilidad, debido a que los quistes de artemia tienen baja digestibilidad, al estar encapsulados (García, 2000).

La tasa de crecimiento específico (TCE), se reduce a medida que avanza la edad y la talla de los organismos (Kaushik, 1995), viéndose influenciada por la proteína de la dieta suministrada con relación a la cantidad y calidad (Boujard, 2001) y factores ambientales, que son altamente variables, como temperatura, oxigeno disponible, amonio, salinidad, fotoperiodo, grado de competencia, entre otros (Moyle \& Cech, 2004).

Según Jauncey (1982), el tipo de alimento proporcionado a los organismos afecta la tasa de crecimiento específico (TCE), lo cual, demarca una importancia significativa como parámetro, en un estudio nutricional, porque puede determinar la calidad proteínica de las dietas.

El alimento suministrado al E. mutisii, en el presente estudio, fue de alto contenido proteico, obteniendo una tasa de crecimiento específico de $5,44 \%$, en peso y $2,95 \%$, en longitud, siendo mayores a las obtenidas en Ictalurus balsanus, que fueron de 2,31\% y 0,56\%, respectivamente (Arce-Uribe \& Luna-Figueroa, 2003); por lo tanto, las dietas altas en contenido proteico en la larvicultura, mejoran las tasas de crecimiento en los individuos.

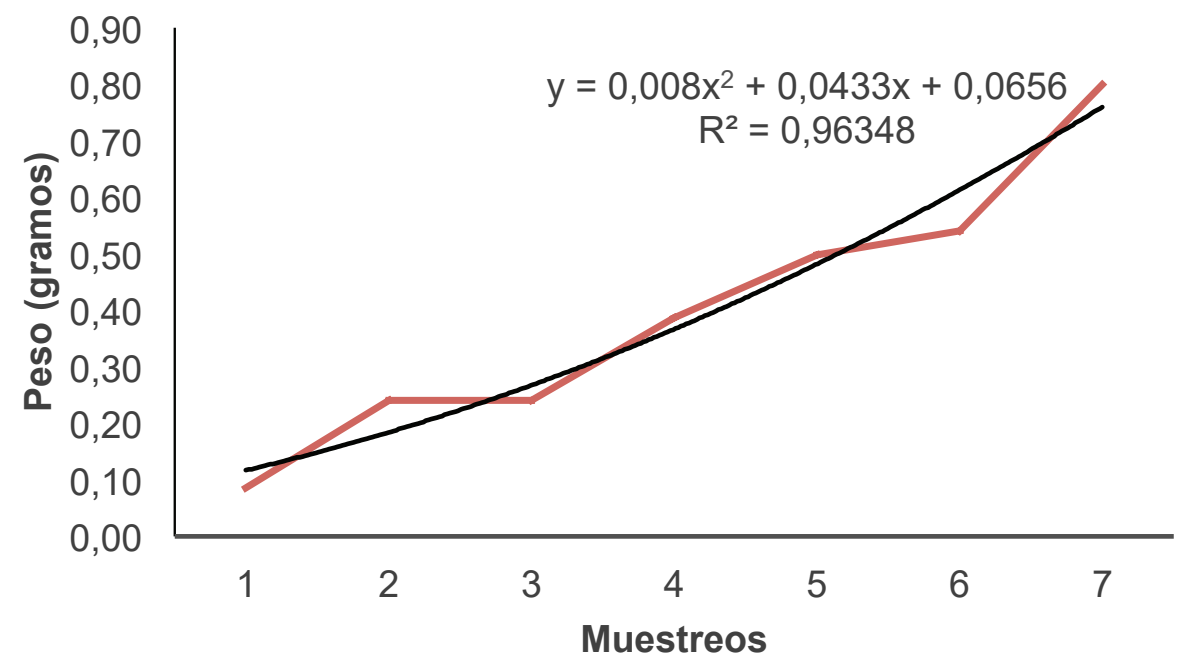

Figura 5. Crecimiento de E. mutisii 47 DPE hasta 77 DPE. 
En ensayos de primera alimentación con Moina sp. y A. salina en bagre asiático Clarias macrocephalus, la sobrevivencia se mantuvo durante 28 DPE por encima de 60\% (Evangelista et al. 2005) y algo similar fue observado en larvas de $P$. fasciatum, alimentadas con zooplancton natural y $A$. salina, hasta los 15 días post-eclosión (DPE) (Núñez et al. 2008). En nuestro caso, la sobrevivencia presentada por E. mutisii fue de $89,5 \%$, en esta etapa de alevinaje, superando a las reportadas anteriormente.
La temperatura a lo largo de todo el ensayo fue de $15,7 \pm 0,4^{\circ} \mathrm{C}$; el lento desarrollo de esta especie, se le atribuye, de manera general, a la temperatura, pues cuando se compara la larvicultura con otras especies de bagres de clima cálido, el tiempo de desarrollo es menor (González Acosta et al. 2017).

Finalmente, en la tabla 1 se muestra un resumen de las etapas de desarrollo para E. mutisii en sus primeras fases de crecimiento, resaltando los eventos más representativos durante este estudio.

Tabla 1. Identificación de etapas del Capitán de la Sabana Eremophilus mutisii en las primeras fases de desarrollo.

\begin{tabular}{|c|c|l|}
\hline ETAPA & DÍA & \multicolumn{1}{|c|}{ EVENTO } \\
\hline \multirow{4}{*}{ Larva } & 1 & nacimiento \\
\cline { 2 - 3 } & 3 & abertura máxima bucal \\
\cline { 2 - 3 } & 9 & $100 \%$ absorción de saco vitelino \\
\cline { 2 - 3 } & 9 & longitud total de $6,88 \mathrm{~mm}$ \\
\hline \multirow{4}{*}{ Post larva } & 11 & inicio de alimentación exógena \\
\cline { 2 - 4 } & 14 & evidencia microscópica y macroscópica de preferencia alimenticia \\
\cline { 2 - 4 } & 46 & sobrevivencia de $58,5 \%$ \\
\hline \multirow{5}{*}{ Alevino } & 47 & co-alimentación (quistes de artemia-alimento artificial) \\
\cline { 2 - 3 } & 50 & suspensión de artemia, solo alimento artificial. \\
\cline { 2 - 3 } & 77 & sobrevivencia de $89,54 \%$ \\
\cline { 2 - 3 } & 77 & ganancia de peso $0,012 \mathrm{~g} /$ día \\
\cline { 2 - 3 } & 77 & tasa de crecimiento específica $5,44 \% \mathrm{~g} /$ día y 2,95\%mm/día \\
\hline
\end{tabular}

Los resultados del presente estudio permiten concluir que la larvicultura en el capitán de la sabana es posible, debido a las altas tasas de sobrevivencia presentadas. Adicionalmente, esta especie presenta un alto de grado de adaptación a las condiciones físicas y químicas del agua, aceptación de alimento, como $A$. salina, principalmente quistes, a partir del 11 DPE y, posteriormente, alimento artificial, desde los 47 DPE.

Por último, al ser este el primer trabajo en describir el desarrollo temprano de individuos producidos en cautiverio, constituye un aporte hacia la obtención e identificación de mecanismos y estrategias, que garanticen el uso y la conservación en cautiverio de E. mutisii, con el fin de fomentar el aprovechamiento acuícola en esta especie, que se encuentra en amenaza de extinción y convertirse en una fuente alimenticia, que contribuya a la seguridad alimentaria de la población Cundiboyacense.

Conflicto de intereses: El manuscrito fue preparado y revisado con la participación de todos los autores, quienes declaramos que no existe conflicto de intereses que ponga en riesgo la validez de los resultados presentados. Financiación: Este estudio fue realizado bajo la cofinanciación del Ministerio de Ciencia, Tecnología e Innovación y la Universidad de Ciencias Aplicadas y Ambientales U.D.C.A, en el proyecto: "Producción en cautiverio del capitán de la sabana, Eremophilus mutisii, con fines de conservación".

\section{REFERENCIAS}

1. ARCE-URIBE, E.; LUNA-FIGUEROA, J. 2003. Efecto de dietas con diferente contenido proteico en las tasas de crecimiento de crias del Bagre del Balsas Ictalurus balsanus (Pisces: Ictaludae) en condiciones de cautiverio. AquaTic. 18:39-47.

2. ARISMENDI, I.; PENALUNA, B.; SOTO, D. 2011. Body condition indices as a rapid assessment of the abundance of introduced salmonids in oligotrophic lakes of southern Chile. Lake and Reservoir Management 27:61-69.

3. ARISTIZABAL, E.O. 2006. Consumo de oxígeno, crecimiento y utilización del alimento durante el desarrollo larval del besugo Pagrus pagrus (Linné 1758) (Pisces: Sparidae). Rev. Biol. Mar. Ocean. 41(2):209-222.

http://dx.doi.org/10.4067/S0718-19572006000200009 
4. ATENCIO-GARCÍA, V.; ZANIBONI-FHILHO, E. 2006. El canibalismo en la larvicultura de peces. Rev. MVZ Córdoba. 11:9-16.

5. BOUJARD, T. 2001. Feeding behaviour and regulation of food intake. p.19-25. En: Guillaume, J.; Kaushik, S.; Bergot, P.; Métailler, R. Nutrition and feeding on fish and crustaceous. Springer and Praxis Publishing, Chichester UK. 408p.

6. EVANGELISTA, A.D.; FORTES, N.R.; SANTIAGO, C.B. 2005. Comparision of some live organism and artificial diet as feed for asian catfish Clarias macrocephalus (Gunther) larvae. J. Appl. Ichthyol. 21(5):427-443. https://doi.org/10.1111/j.1439-0426.2005.00643.x

7. FALK-PETERSEN, I.B. 2005. Comparative organ differentiation during early life stages of marine fish. Fish Shellfish Immunol. 19(5):397-412. https://doi.org/10.1016/j.fsi.2005.03.006

8. FAO. 2009. Oncorbynchus mykiss. In: Cultured aquatic species fact sheets. Text by Cowx, I.G. Edited and compiled by Valerio Crespi and Michael New. CD-ROM (multilingual). Disponible desde Internet en: http://www.fao.org/tempref/FI/CDrom/aquaculture/ $\mathrm{I} 1129 \mathrm{~m} /$ file/es/es_rainbowtrout.htm (con acceso 09/06/18).

9. GARCÍA, A. 2000. Valor nutricional de los quistes de Artemia y su uso como fuente de proteína en dietas artificiales para larvas de peces In: Cruz -Suárez, L.E., Ricque-Marie, D., Tapia-Salazar, M., Olvera-Novoa, M.A. y Civera-Cerecedo, R. (Eds.). Avances en Nutrición Acuícola V. Memorias del V Simposium Internacional de Nutrición Acuícola. 19-22 Noviembre, 2000. Mérida, Yucatán. p.287-299.

10. GARCÍA-GASCA, A.; GALAVIZ, M.; GUTIÉRREZ, J.; GARCIA-ORTEGA, A. 2006. Development of the digestive tract, trypsin activity, and gene expression in eggs and larvae of the bullseye puffer fish Sphoeroides annulatus. Aquaculture. 251(2-4):366-376.

https://doi.org/10.1016/j.aquaculture.2005.05.029

11. GISBERT, E.; MOREIRA, C.; CASTRO-RUIZ, D.; ÖZTÜRK, S.; FERNÁNDEZ, C.; GILLES, S.; NUÑEZ, J.; DUPONCHELlE, F.; TELLO, S.; RENNO, J.F.; GARCÍA-DÁVILA, C.; DARIAS, M.J. 2014. Histological development of the digestive system of the Amazonian pimelodid catfish Pseudoplatystoma puncifer. Animal. 8(11):1765-1776.

https://doi.org/10.1017/S1751731114001797

12. GONZÁLEZ ACOSTA, J.A.; ZAPATA BERRUECOS, B.E.; ROSADO PUCCINI, R. 2017. Desarrollo embrionario del capitan de la sabana, Eremophilus mutisii (Pisces:
Trichomycteridae). Rev. Medicina Veterinaria. 35:137-147. http://dx.doi.org/10.19052/mv.4396

13. GONZÁLEZ-ACOSTA, J.A.; ROSADO-PUCCINI, R. 2005. Reproducción Inducida con hormonas en Eremophilus mutisii Humboldt, 1805 (Pisces: Trichomycteridae) en Guasca Cundinamarca, Colombia. Revista de Investigación. 5(2):233-240.

14. GORE, S.R. 2006. Nutritional support of fish. J. Exotic Pet Medicine. 15(4):264-268. https://doi.org/10.1053/j.jepm.2006.09.005

15. HAMRE, K.; SRIVASTAVA, A.; RONNESTAD, I.; MANGORJENSEN, A.; STOSS, J. 2008. Several micronutrients in the rotifer Brachionus sp. may not fulfil nutricional requeriments of marine fish larvae. Aquaculture Nutrition. 14(1):51-60. https://doi.org/10.1111/j.1365-2095.2007.00504.x

16. IDEAM. 2016. Instituto de Hidrología, Meteorología y Estudios Ambientales. Disponible desde Internet en: http://www.ideam.gov.co/ (con acceso 25/10/2016).

17. JAUNCEY, K. 1982. The effects of varying dietary protein level on the growth, food conversion, protein utilization and body composition of juvenile tilapias (Sarotherodon mossambicus). Aquaculture. 27(1):43-54. https://doi.org/10.1016/0044-8486(82)90108-9

18. KAUSHIK, S.J. 1995. Nutrient requeriments, supply and utilization in the context of carp culture. Aquaculture. 129(1-4):225-241. https://doi.org/10.1016/0044-8486(94)00274-R

19. KESTEMONT, P.; JOURDAN, S.; HOUBART, C.; PASPATIS, M.; FONTAINE, P.; CUVIER, A.; KENTOURI, M.; BARAS, E. 2003. Size heterogeneity, cannibalism and competition in cultured predatory fish larvae: biotic and abiotic influences. Aquaculture. 227:333-356. https://doi.org/10.1016/S0044-8486(03)00513-1

20. LANDINES, M.A.; PRIETO, C.A.; RODRIGUEZ, L.; ROSADO, R. 2017. Perfil de esteroides sexuales del capitán de la sabana (Eremophilus mutisii) durante un ciclo hidrológico completo. Rev U.D.C.A Act \& Div Cient. 20(1):43-50. https://doi.org/10.31910/rudca.v20.n1.2017.61

21. LEMUS-PORTILLO, C.; ECHAVARRÍA-ECHAVARRÍA, M.C.; ROJAS, J.E.; ALVAREZ-DIAZ, J.E.; LEONPARDO, K.J.; AGUILAR-ORJUELA, S.; MALDONADO, J. 2020. Estado de conservación y distribución del pez de agua dulce Capitán de la Sabana Eremophilus mutisii en Cundinamarca, Colombia. Actualidades Biológicas. 42(112). https://doi.org/10.17533/udea.acbi.v42n112a02 
22. LEMUS-PORTILLO, C.; MOJICA CORZO, J.I.; ECHAVARRÍA, M.; AVELLA CASTIBLANCO, C.; LÓPEZ MENESES, W.F.; USECHE LOSADA, P.; MOJICA, I. 2017. Programa nacional para la conservación de la especie endémica de Colombia pez capitán de la sabana (Eremophilus mutisii). Ministerio de Ambiente y Desarrollo Sostenible; Secretaría Distrital de Ambiente; Universidad Manuela Beltrán. 60p.

23. LI, S.; MATHIAS, J. 1982. Causes of high mortality among cultured larval walleyes. Transactions of the American Fisheries Society. 111(6):710-721.

https://doi.org/10.1577/1548-8659(1982)111<710:COH $\mathrm{MAC}>2.0 . \mathrm{CO} ; 2$

24. LÓPEZ-LÓPEZ, V.V.; RODRÍGUEZ M., G.A.; GALAVIZ, M.A.; ROMAN REYES, C.; MEDINA HERNÁNDEZ, E.; DABROWSKI, K.; HAWS, M.C. 2015. Descripción histológica comparativa del desarrollo del sistema digestivo y visual de larvas de chame Dormitator latifrons (Pisces: Eleotridae). Lat. Am. J. Aquat. Res. 43(3):484-494. http:/ /dx.doi.org/10.3856/vol43-issue3-fulltext-10

25. MARCIALES CARO, L.J.; DIAZ-OLARTE, J.J.; MEDINAROBLES, V.M.; CRUZ-CASALLAS, P.E. 2010. Evaluación del crecimiento y sobrevivencia de larvas de bagre rayado Pseudoplatystoma fasciatum (Linneaus, 1766) alimentadas con alimento vivo natural y enriquecido con ácidos grasos. Rev. Col. Cienc. Pecua. 23:308-316.

26. MOJICA, I.; CASTELLANOS, C.; USMA, S.; ÁLVAREZ, R. (Eds). 2002. "Libro rojo de peces dulceacuícolas de Colombia". Serie Libros Rojos de Especies Amenazadas de Colombia. Instituto de Ciencias Naturales Universidad Nacional de Colombia, Ministerio del Medio Ambiente, Bogotá, Colombia.

27. MOJICA, I.; USMA, S.; ÁlVAREZ, R.; LASSO, C. (Eds). 2012. "Libro rojo de peces dulceacuícolas de Colombia". Serie Libros Rojos de Especies Amenazadas de Colombia. Instituto de Investigación de Recursos Biológicos Alexander von Humboldt, Ministerio del Medio Ambiente, Bogotá, Colombia.

28. MONCALEANO, E.; SÁNCHEZ, C.; PRIETO, C. 2018. Estudio histologico y morfologico del desarrollo embrionario del pez Capitan de la sabana (Eremophilus mutisii). Rev. U.D.CA Act. \& Div. Cient. 21(2):479-489. https://doi.org/10.31910/rudca.v21.n2.2018.1073

29. MORENO, J.M.; AGUILAR, F.A.; BOADA, N.S.; ROJAS, J.; PRIETO, C. 2019. Análisis morfométrico e índices corporales del capitán de la sabana. Rev. Facultad de Medicina Veterinaria y de Zootecnia. 66(2):141-153. https://dx.doi.org/10.15446/rfmvz.v66n2.82433
30. MOYLE, P.B.; CECH, J.J. 2004. Fishes: An introduction to Ichthyology. Prentice Hall (Uppersaddle River, .NJ USA). $726 \mathrm{p}$.

31. NÚÑEZ, J.; DUGUÉ, R.; CORCUY, N.; DUPONCHELLE, F; RENNO, J.; RAYNAUD, T.; HUBERT, N.; LEGENDRE, M. 2008. Induced breeding and larval rearing of Surubí,Psendoplatystoma fasciatum (Linnaeus, 1766), from the Bolivian Amazon. Aquaculture Res. 39(7):764-776. https://doi.org/10.1111/j.1365-2109.2008.01928.x

32. QUANTZ, G. 1985. Use of endogenous energy sources by larval turbot Scophthalmus maximus. Trans. Am. Fish. Soc. 114:558-563.

https://doi.org/10.1577/1548-8659(1985)114<558:UOE $\mathrm{ESB}>2.0 . \mathrm{CO} ; 2$

33. ROSADO, R. 2007. Aspectos reproductivos y piscícolas. En: Rosado, R.; Alvarez, R.; González, J.; Surárez, R. Aspectos reproductivos y piscícolas. Editorial Universidad del Rosario (Bogotá). p.51-61.

34. SORIANO SALAZAR, M.; HERNÁNDEZ OCAMPO, D. 2002. Tasa de crecimiento del Pez Ángel Pterophyllum scalare (Perciformes: Cichlidae) en condiciones de laboratorio. Acta Universitaria. 12(2):28-33. https://doi.org/10.15174/au.2002.277

35. TACON, A. 1987. The nutrition and feeding of farmed fish and farmed fish and shrimp- a training manual. The essential nutrients. FAO. Trust Fund GCO/ RLA/075/ITA. Brasilia, Brasil. 117p.

36. TORRES-TABARES, A.; VELASCO-SANTAMARÍA, Y.M.; RAMÍREZ-MERLANO, J.A. 2014. Características morfológicas, morfométricas, merísticas y de manejo de la primera alimentación de larvas de escalar altum (Pterophyllum altum) (Pellegrin, 1903). Orinoquia. 18(2):183-192. https://doi.org/10.22579/20112629.374

37. VALBUENA V., R.; ZAPATA-BERRUECOS, B.; OTEROPATERNINA, A. 2013. Evaluación de la primera alimentación en larvas de capaz Pimeludus grosskopfii bajo condiciones de laboratorio. Rev. MVZ Córdoba. 18(2):3518-3524. https://doi.org/10.21897/rmvz.176

38. VALDERRAMA, M.; MORALES-BETANCOURT, M.; HERNÁNDEZ, S. 2011. Eremophilus mutisii. En: Lasso, A.E.; Agudelo Cordoba, L.F., Jimenez-Segura, H.; RamirezGil, M.; Morales-Betancourt, R.E.; Ajiaco-Martínez, F.; de Paula Gutierrez, F.; Usma Oviedo, J.S.; Muñoz Torres, S.E.; Sanabria Ochoa, A.L. (Eds). Catálogo de los recursos pesqueros continentales de Colombia. Serie Editorial Recursos Hidrobiológicos y Pesqueros Continentales 
de Colombia. Instituto de Investigación de Recursos Biológicos Alexander von Humboldt. Bogotá, D.C., Colombia, $715 p$

39. WILLIAMS, K.; PAPANIKOS, N.; PHELPS, R.P.; SHARDO, J.D. 2004. Development, growth and yolk utilization of hatchery-reared red snapper Lutjanus campechanus larvae. Mar. Ecol. Prog. Ser. 275:231-239.

https://doi.og/10.3354/meps275231 